\title{
A numerical investigation into the plastic buckling of circular cylinders
}

\author{
R. Shamass \& G. Alfano \\ Brunel University, Uxbridge, UK \\ F. Guarracino \\ University of Naples "Federico II", Naples, ITALY
}

\begin{abstract}
It has been long known that finding a thoroughly good agreement with experimental results for the buckling loads of circular cylinders in the plastic range constitutes a very difficult task, beyond the same as well challenging problem in the elastic range. This paper attempts to contribute to the understanding of this issue by discussing a few numerical examples in comparison with some classical experimental results reported by Blachut et al. (1996) and Batterman (1965). It is shown that finite-element analyses grounded on the hypoelasticity-based extension of small-strain theory of plasticity to geometrically nonlinear problems lead to good agreement with experimental results, although in some cases the sensitivity to the amount and shape of imperfection plays an important role, a fact which leaves open issues in terms of fully predictive capability of the computational models.
\end{abstract}

\section{INTRODUCTION}

Imperfection sensitive structures are characterized by the fact that when the structure undergoes buckling its load carrying capacity diminishes and the buckling load is theoretically the maximum load that can be supported (Hutchinson, 1972). As a result, small imperfections have an appreciable effect on the maximum actual load that these structures can support and this is particularly true for circular cylindrical shells in the plastic range (Bushnell, 1982).

On these bases the present work attempts to contribute to a further understanding of this problem by means of a few numerical examples and comparisons with some classical results (Batterman 1965, Blachut et al. 1996). It is shown that using a geometrically nonlinear shell-based finite-element formulation and an extension of the small-strain elastoplasticity theory based on linear hypo-elastic relations between stress and elastic strain rates, a good agreement between numerical and experimental results can be obtained.

\section{BRIEF OUTLINE OF THE ADOPTED NUMERICAL APPROACH}

\subsection{FE modelling}

When buckling occurs during the loading process, even if the stress-strain relation remains linear, large deformations and the change in the geometry cause the structure to behave non-linearly and the analysis requires a non-linear geometric approach. Additionally, for the vast majority of moderately thick shells, the bifurcation load occurs mostly in the plastic range of the material and an adequate representation of the material behaviour must be introduced in the analysis.

The commercially available Finite Element package ABAQUS provides tools to carry out all these kinds of non-linear analyses by means of a modified version of the arc-length method first proposed by Riks (1979). The Modified Riks method is able to identify the limit points and trace the equilibrium path by using both displacement increments and the scalar load increment as unknown variable in the iteration scheme. The methods falls within the general category of the linearized arc-length methods and searches the solution in the generalized plane orthogonal to the tangent line to the current iterative tentative solution, see for example Crisfeld (1991).

\subsection{Description of imperfections}

Imperfections are accounted in the geometry of the problem by adding the form of a suitable buckling mode or the combination of multiple linear eigenmodes. This is practically achieved by scaling and adding buckling eigenmodes to a perfect geometry in order to create a perturbed model. The scale number is usually taken as a percentage of the shell thickness, $t$. 


\subsection{Material modeling}

Simulations were conducted adopting a constitutive model based on the $J_{2}$ theory of plasticity and a von Mises yield criterion with nonlinear isotropic hardening extended to the large-strain regime by using spatial stress and strain measures and a hypoelastic relation between the rates of stress and elastic strain. Starting from an additive decomposition of the spatial rates of the deformation tensor into its elastic and plastic parts,

$\mathbf{d}=\mathbf{d}_{e}+\mathbf{d}_{p}$

the rate of the Cauchy stress tensor $\dot{\sigma}$ is obtained from the elastic part of the strain tensor through the isotropic linear elastic relation:

$\dot{\boldsymbol{\sigma}}=2 G \mathbf{d}_{e}+\mu \operatorname{trd} \mathbf{d}_{e} \mathbf{1}$

where $G$ and $\mu$ are the shear elastic modulus and the second Lamé's constant and $\mathbf{1}$ is the rank-2 identity tensor. The von Mises' yield function $f$ is then introduced as follows:

$$
f(\boldsymbol{\sigma})=\|\operatorname{dev}(\boldsymbol{\sigma})\|-\sqrt{\frac{2}{3}} \bar{\sigma}\left(\varepsilon_{p}^{e q}\right)
$$

where $\operatorname{dev}(\boldsymbol{\sigma})$ denotes the deviatoric part of the Cauchy stress tensor and $\bar{\sigma}$ represents the uniaxial yield strength which, in order to model nonlinear isotropic hardening, is assumed to be an increasing function of the equivalent plastic strain $\varepsilon_{p}^{e q}$ defined at time $t$ by

$$
\varepsilon_{p}^{e q}=\int_{0}^{t}\left\|\dot{\boldsymbol{\varepsilon}}_{p}(\tau)\right\| d \tau
$$

The evolution of the plastic strain tensor is given by the flow rule:

$$
\dot{\boldsymbol{\varepsilon}}_{p}=\dot{\lambda}\left(\frac{\partial f}{\partial \mathbf{s}}\right)_{\mathbf{s}=\operatorname{dev}(\boldsymbol{\sigma})}
$$

where $\dot{\lambda}$ is related to the yield function through the Kuhn-Tucker conditions:

$$
\dot{\lambda} \geq 0 \quad f(\boldsymbol{\sigma}) \leq 0 \quad \dot{\lambda} f(\boldsymbol{\sigma})=0
$$

The above described constitutive relation has been the subject of controversial debate because relation (2) can be shown to be non hyper-elastic, and leads to fictitious numerical dissipation. However, this formulation is widely implemented in many of the commercial codes, including ABAQUS, and it can be argued that the hypo-elasticity of the formulation has limited influence on the results because, even when strains are large, the elastic part of the strain is typically still very small and therefore close to the limit where hypo-elastic and hyper-elastic formulations coincide (Simo and Hughes, 1998).

\section{CIRCULAR CYLINDERS UNDER AXIAL TENSION AND EXTERNAL PRESSURE}

\subsection{Geometry, boundary conditions, material properties and loading}

Blachut et al. (1996) conducted experiments on 30 mild-steel machined cylinders, $\approx 34 \mathrm{~mm}$ in diameter with a length-diameter ratio (LD) of 1.0, 1.5 and 2.0. In the present study twelve of these cylinders were chosen for the numerical analysis. In the experimental setting one flange of the specimen was rigidly attached to the end flange of the pressure chamber and the other flange was bolted to a coupling device which in turn was bolted to the load cell, see Figure 1. The load cell was centered with respect to the test chamber in order to prevent any eccentricity of the axial load applied to the cylindrical specimen.
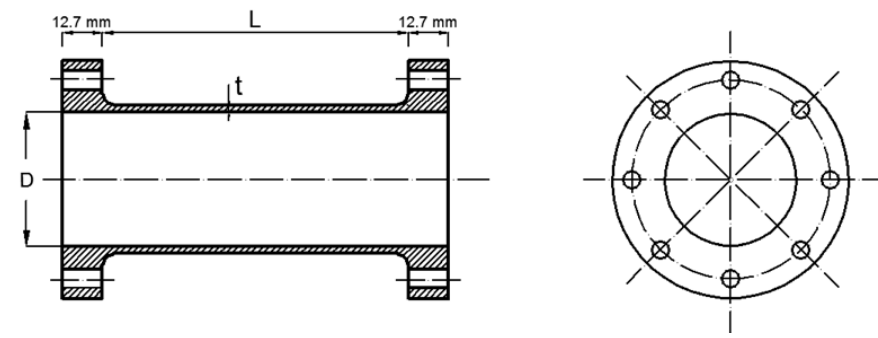

Figure 1. Experimental setting (Blachut et al. 1996).

\subsection{FE analyses}

In the FE model a reference point was located at the centroid of the top end of the cylinder and kinematic coupling constraints were assigned to it. The axial load was applied directly to the reference point. One end of the cylinder was clamped, while the nodes at the other end were allowed displacements in the axial direction, see Figure 2

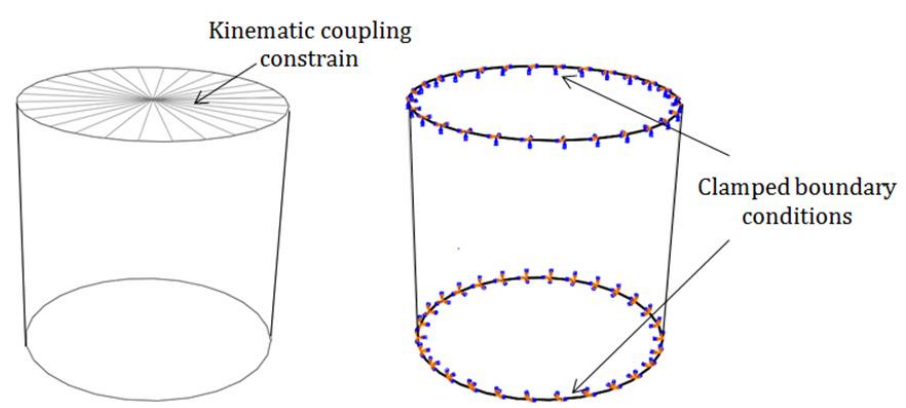

Figure 2. FE modeling of Blachut experiments.

In order to simulate the experimental settings, two loading conditions were considered: an axial load in the longitudinal direction of the cylinder and a pressure applied normally to the surface of the shell elements. The material properties were derived from the material tests conducted by Blachut et al. (1996). Young's modulus, E, and Poisson's ratio, $v$, were set equal to $212 \mathrm{GPa}$ and 0.31 , respectively. The yield 
stress of the mild steel was assumed to be $328 \mathrm{MPa}$ and uniform in the model

In Table 1 the predicted buckling pressures provided by the numerical simulations are compared with the experimental findings.

Table 1. Experimental (Blachut et al. 1996) vs numerical predictions

\begin{tabular}{|c|c|c|c|c|}
\hline \multirow{3}{*}{ Spec. } & \multirow{3}{*}{$\begin{array}{l}\text { Axial } \\
\text { tension } \\
\text { (N) }\end{array}$} & \multicolumn{2}{|c|}{$\begin{array}{l}\text { External pressure experimental } \\
(\mathrm{Mpa})\end{array}$} & \multirow{3}{*}{ Pexp/Pnum } \\
\hline & & $\begin{array}{c}\text { Experimental re- } \\
\text { sults (Blachut et } \\
\text { al.,1996) }\end{array}$ & $\begin{array}{l}\text { Numerical } \\
\text { results }\end{array}$ & \\
\hline & & Pexp & Pnum & \\
\hline S1 & 17960 & 4.07 & 5.43 & 0.75 \\
\hline S2 & 0 & 12.76 & 13.14 & 0.97 \\
\hline S3 & 18000 & 4 & 4.91 & 0.81 \\
\hline S4 & 3990 & 10.41 & 11.79 & 0.88 \\
\hline S5 & 12010 & 8.28 & 8.72 & 0.95 \\
\hline S6 & 15030 & 6.55 & 7.72 & 0.85 \\
\hline S7 & 7970 & 9.66 & 10.39 & 0.93 \\
\hline M2 & 10670 & 8.14 & 7.79 & 1.04 \\
\hline M12 & 18530 & 4.48 & 4.16 & 1.08 \\
\hline$M 7$ & 15060 & 5.52 & 5.64 & 0.98 \\
\hline$L 4$ & 8210 & 10.34 & 9.90 & 1.04 \\
\hline$L 8$ & 16490 & 5.17 & 6.24 & 0.83 \\
\hline
\end{tabular}

In Figures 3-5, the load-displacement curves obtained for all the simulations are shown. They clearly reveal the unstable nature of the post-buckling regime, but also show that extent of structural softening is significantly influenced by the different values of the axial pressure.

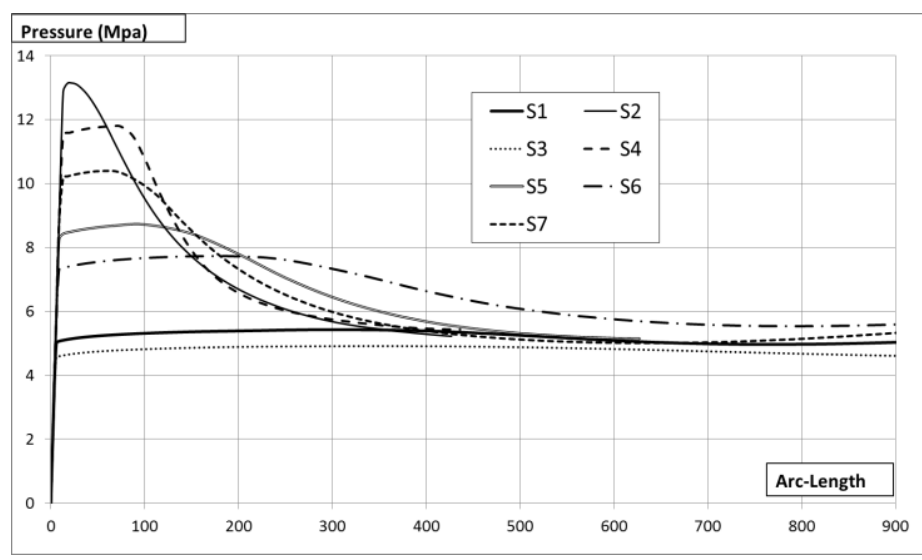

Figure 3. Load-displacement curves numerically determined for specimens S1-S7.

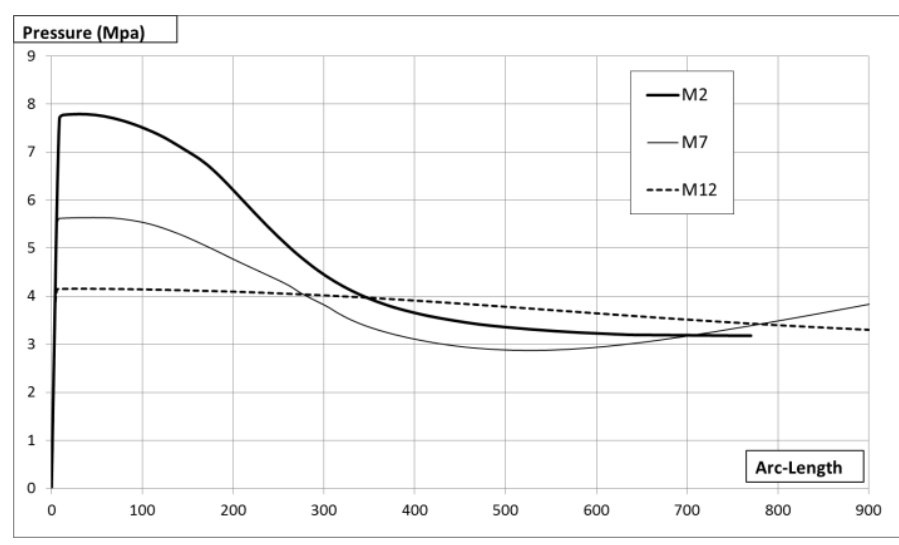

Figure 4. Load-displacement curves numerically determined for specimens S2, M7 and M12.

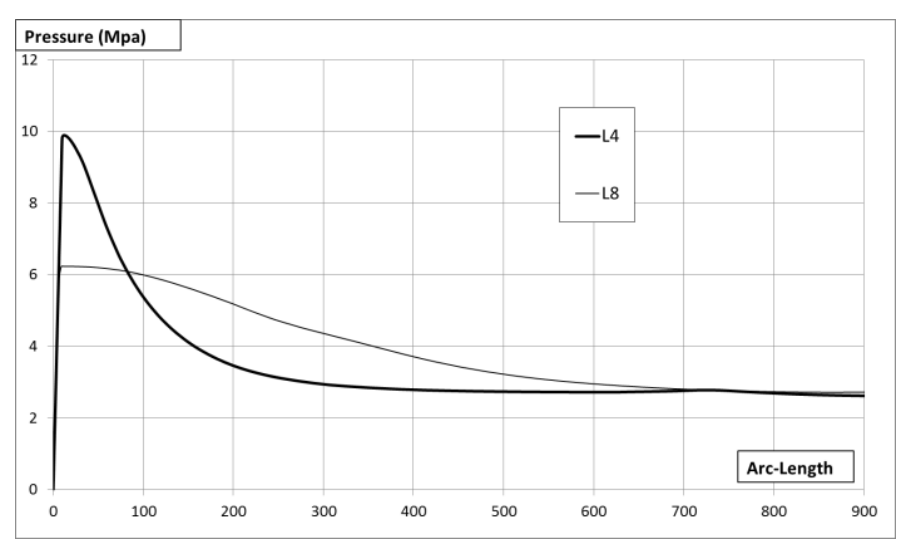

Figure 5. Load-displacement curves numerically determined for specimens L4 and L8.

\section{CIRCULAR CYLINDERS UNDER AXISYMMETRIC COMPRESSION LOAD}

\subsection{Geometry, boundary conditions, material properties and loading}

Batterman (1965) used tubular specimens made of aluminum alloy 2024-T4, clamped at both ends as in the case of Blachut tests. By applying axisymmetric compression, Batterman tested 30 cylinders with a radius-thickness ratio $(\mathrm{R} / \mathrm{t})$ between 9.7 and 121.25 with great emphasis on the buckling pattern of each cylinder. Nine cylindrical shells were chosen for the present numerical analysis

\subsection{FE analyses}

The material characterization for the FE analyses was derived from the test results from Batterman. Young's modulus, E, Poisson's ratio, v, hardening parameter, n, yield stress, $\sigma y$, and yield offset $\alpha$ of the cylindrical shell were taken as $74.46 \mathrm{GPa}, 0.32,14.45,389.55$ and 0.382 , respectively. 


\section{CONCLUSIONS}

Table 2. Experimental (Battermann 1965) vs ABAQUS results

\begin{tabular}{|c|c|c|c|}
\hline Spec. & $\begin{array}{c}\text { Sexp=Experimental } \\
\text { Buckling Stress } \\
\text { (Batterman, 1965) }\end{array}$ & $\begin{array}{c}\text { Snum=Numerically } \\
\text { predicted buckling } \\
\text { stress }\end{array}$ & Sexp/Snum \\
\hline 12 & 480.08 & 455.57 & 1.05 \\
\hline 18 & 482.63 & 454.87 & 1.06 \\
\hline 22 & 439.8 & 433.18 & 1.02 \\
\hline 5 & 410.72 & 401.56 & 1.02 \\
\hline 15 & 382.6 & 377.44 & 1.01 \\
\hline 16 & 354.25 & 366.92 & 0.97 \\
\hline 26 & 301.23 & 307.92 & 0.98 \\
\hline 8 & 227.73 & 220.93 & 1.03 \\
\hline 9 & 219.05 & 234.04 & 0.94 \\
\hline
\end{tabular}

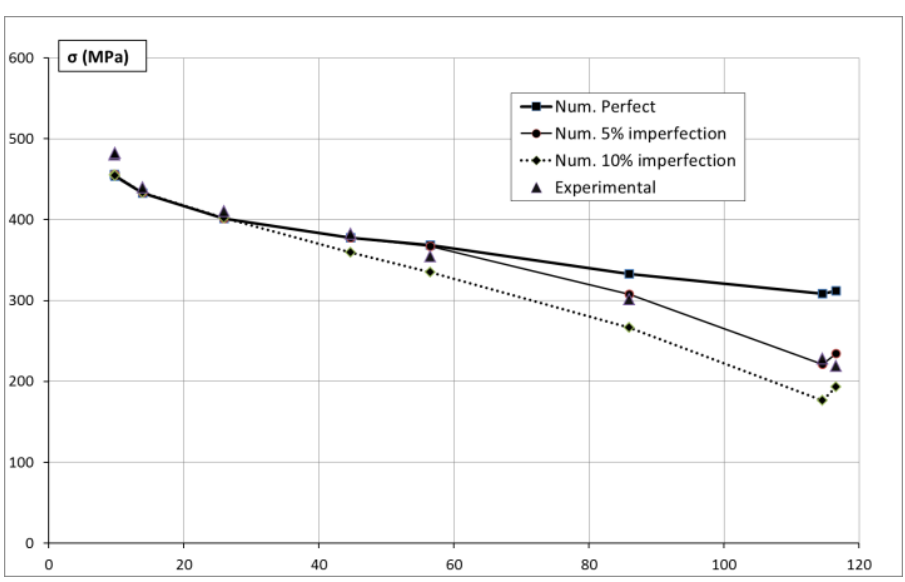

Figure 6. Comparison between numerical and experimental ratios for different imperfection ratios.

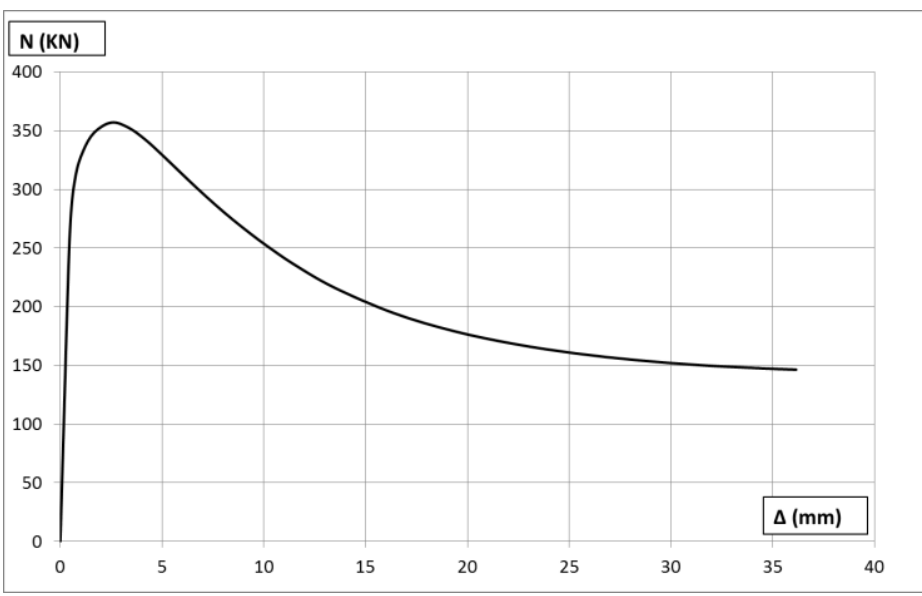

Figure 7. Load displacement curve obtained for specimen 12.

Figure 6 shows the influence of the imperfection ratio on the results of the nonlinear post-buckling analysis and reveals that such sensitivity increases with increasing values of the ratio between the outer radius, $R$, and the thickness, $t$, of the specimen. Figure 7 shows a typical load-displacement curve numerically determined.
Nonlinear post-buckling analyses which properly account for the plastic response of the material have been found to provide very good agreement with some classical experimental results reported in the literature.

The importance of a correct modeling of loading, nonlinearity and boundary conditions (see, for example, Pietraszkiewicz 1984 and Guarracino 2007) has been once again confirmed and the results are shown to be sensitive to the amount imperfection used, as it is expected for this kind of structures. This is particularly so for moderately thick cylinders, a case for which the influence of plasticity is fairly noticeable. The results suggest that the use of a hypo-elasticity based extension of the small-strain elasto-plasticity von Mises model to the case of (moderately) large strains is adequate, despite the theoretical issues raised by the lack of preservation of the elastic energy within a closed cycle.

The presented results are however very preliminary and the undertaken research aims at thoroughly examining the influence of different modeling assumptions which can be used to represent material and geometrical nonlinearities, of different finiteelement approximations of the boundary value problem and, finally, of various solution approaches.

\section{REFERENCES}

Batterman, S.C. 1965. Plastic buckling of axially compressed cylindrical shells. AIAA Journal 3 (2): 316-325.

Blachut, J., Galletly, G. \& James, S. 1996. On the plastic buckling paradox for cylindrical shells. Proc Instn Mech Engnrs 210 (5): 477-488.

Bushnell, D. 1982. Plastic buckling. In S.Y. Zamrik \& D. Dietrich (eds), Pressure vessels and piping; design technology1982: a decade of progress: 47-117. New York: ASME.

Crisfeld M.A. 1991. Non-linear Finite Element Analysis of Solids and Structures, vol. 1. Wiley, Chichester.

Guarracino F. 2007. Considerations on the numerical analysis of initial post-buckling behaviour in plates and beams. ThinWall Struct 45: 845-848.

Hutchinson, J.W. 1972. On the postbuckling behavior of imperfection-sensitive structures in the plastic range. Journal of Appl. Mech. 39: 155-162.

Pietraszkiewicz, W. 1984. Lagrangian description and incremental formulation in the non-linear theory of thin-shells. Int. J. Non-Linear Mechanics 19 (2): 115-140.

Riks, E., 1979. An incremental approach to the solution of snapping and buckling problems. Int Journal Solids Struct 15:529-551.

Simo, J.C. \& Hughes, T.J.R. 1998. Computational Inelasticity. Springer, Berlin. 